\title{
Rabaska
}

Revue d'ethnologie de l'Amérique française

\section{Chaire de recherche du Canada sur l'identité métisse (CRCIM) (Université de Saint-Boniface)}

\section{Denis Gagnon}

Volume 10, 2012

URI : https://id.erudit.org/iderudit/1013589ar

DOI : https://doi.org/10.7202/1013589ar

Aller au sommaire du numéro

Éditeur(s)

Société québécoise d'ethnologie

ISSN

1703-7433 (imprimé)

1916-7350 (numérique)

Découvrir la revue

Citer ce document

Gagnon, D. (2012). Chaire de recherche du Canada sur l'identité métisse

(CRCIM) (Université de Saint-Boniface). Rabaska, 10, 340-341.

https://doi.org/10.7202/1013589ar 


\section{Ouest}

\section{Chaire de recherche du Canada sur l'identité métisse (CRCIM)}

Université de Saint-Boniface

200, avenue de la Cathédrale

Saint-Boniface (Manitoba)

$\mathrm{R} 2 \mathrm{H} 0 \mathrm{H} 7$
Téléphone : (204) 237-1818, poste 454

Courriel : degagnon@ustboniface.mb.ca

\section{Faits saillants}

L'événement le plus important de l'année 2010-2011 a été la publication aux Presses de l'Université Laval de l'ouvrage collectif L'Identité métisse en question : stratégies identitaires et dynamismes culturels, avec la collaboration d'Hélène Giguère. Trois de mes étudiants au doctorat, Joanna Seraphim, Fabien Tremblay et Emmanuel Michaux, sont parmi les douze auteurs de ce collectif auquel j'ai collaboré avec la « chaire de recherche du Canada sur l'identité métisse et les études métisses au Canada »et par la rédaction de l'introduction avec Hélène Giguère «Le métissage : un processus identitaire incontournable, des enjeux négligés ».

Soulignons également la publication d'un article dans la revue Anthropologie et sociétés, «Identité trouble et agent double. L'ontologie à l'épreuve du terrain », vol. $35, \mathrm{n}^{\circ} 3$, p. 147-165.

\section{Collaborations}

Au sujet des collaborations, je poursuis ma collaboration avec Léonard Rivard pour le volet sauvegarde et mise en valeur du patrimoine culturel et linguistique des Métis francophones dans le cadre de la subvention de l'ARUC sur les identités francophones de l'Ouest à l'Université de Saint-Boniface. Je poursuis également ma collaboration avec Robert Papen (UQÀM) dans le cadre des travaux de la chaire et de l'ARUC, et je suis toujours membre régulier du Centre interuniversitaire d'études et de recherches autochtones (CIÉRA) de l'Université Laval où je suis également professeur associé au département d'anthropologie. Enfin, je collabore pour une deuxième année avec la chercheuse Sigrid Norris au projet World-Wide Project on Personnal Identity du Multimodal Research Center qu'elle dirige à l'Université d'Auckland en NouvelleZélande.

\section{Recherche}

En décembre 2011, j'ai été chercheur étranger invité à l'Institut Marcel-Mauss de l'École des hautes études en sciences sociales à Paris où j'ai présenté un séminaire sur le métissage religieux entre chamanisme et catholicisme, et où j'ai effectué une recherche exploratoire à la Bibliothèque nationale de France. En mai 2012, j'ai donné une conférence publique intitulée « Métissage et identité / Regards croisés CanadaJapon » à l'Institut franco-japonais du Kansai à Kyoto au Japon. J'ai supervisé le travail de deux stagiaires postdoctorales : Annik Chiron de la Casinière a produit un rapport de recherche « Pardon pour les pensionnats : encore un combat pour les Métis de l'Ouest canadien » qui sera publié aux Presses de l'Université Laval ; et 
Estelle Cambe qui a fait un rapport sur la construction identitaire des Métis dans la littérature de l'Ouest canadien. J'ai entrepris plusieurs collaborations internationales sur le métissage avec les chercheurs suivants : un doctorant de l'Institut d'histoire universelle de l'Académie des sciences de Russie qui étudie les Métis de Yakoutie en Sibérie ; une doctorante de Marseille qui travaille sur les populations métissées de cette ville; et une étudiante de $1^{\text {er }}$ cycle en Belgique qui travaille sur le métissage congo-belge.

Denis Gagnon

\section{Centre canadien de recherche sur les francophonies en milieu minoritaire} Institut français

Université de Régina

3737 Wascana Parkway

Régina (Saskatchewan) S4S 0A2

Téléphone : (306) 337-3273

Télécopieur : (306) 585-5183

Courriel :crfm.if@uregina.ca

Le Centre canadien de recherche sur les francophonies en milieu minoritaire (CRFM) de l'Institut français (Université de Régina) est un centre de recherche original, s'inscrivant dans une démarche pionnière d'engagement université-communauté. Fondé en 2005, le CRFM dirige, de façon multidisciplinaire et interinstitutionnelle, un programme de recherche novateur qui porte en priorité sur l'expérience de la communauté fransaskoise, tout en s'ouvrant à celle d'autres francophonies, au niveau canadien et international. Le CRFM favorise également des recherches qui permettent de comparer cette expérience francophone à celle d'autres groupes ethnoculturels qui cherchent à s'affirmer en milieu minoritaire. Dominique Sarny a exercé la fonction de directeur du CRFM jusqu'au mois de juin 2012 ; Sheila Petty a pris sa succession

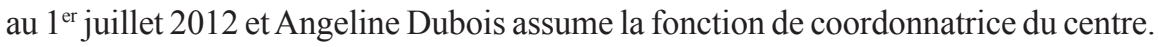

\section{Conférences de l'Institut}

- "Portrait de l'engagement francophone en contexte minoritaire », conférence retransmise en direct de la Faculté des sciences sociales de l'Université d'Ottawa, le 24 janvier 2012, par Éric Forgues, Institut canadien de recherche sur les minorités linguistiques, Université de Moncton. Cette conférence était ouverte au public, dans notre salle équipée du système de vidéoconférence.

- «Enjeux et défis de l'immigration francophone en Saskatchewan », le 22 mars 2012, par Bruno Dupeyron, École d'études supérieures de politiques publiques JohnsonShoyama (Université de Régina et University of Saskatchewan) et chercheur associé à la Chaire de recherche en immigration, ethnicité et citoyenneté (CRIEC) à l'Université du Québec à Montréal (UQÀM). Cette conférence a été organisée dans le cadre d'une soiréedébat «La fransaskoisie face aux défis de l'immigration », présidée par Dominique Sarny, directeur du CRFM et ethnologue lors des «Rendez-vous de la Francophonie » de l'Institut français. Une table ronde a permis de poursuivre le débat, en présence de membres de la communauté, de nouveaux arrivants et de Bruno Dupeyron. - « Les Tribunaux et l'arbitrage en Nouvelle-France et au Québec de 1740 à 1784 ». 